\title{
Attitudes and Practices of a Sample of Nova Scotian Physicians for the Implementation of HIV Pre-Exposure Prophylaxis
}

This article was published in the following Dove Press journal: HIVIAIDS - Research and Palliative Care

\author{
Jad Sinno $\mathbb{D I}^{1,2}$ \\ Nicole Doria' \\ Nicholas Cochkanoff' \\ Matthew Numer' \\ Heather Neyedli' \\ Darrell Tan (D) ${ }^{2,3}$ \\ 'School of Health and Human \\ Performance, Dalhousie University, \\ Halifax, NS, Canada; ${ }^{2}$ Dalla Lana School \\ of Public Health, University of Toronto, \\ Toronto, ON, Canada; ${ }^{3}$ Department of \\ Medicine, St. Michael's Hospital, Toronto, \\ ON, Canada
}

Introduction: Pre-exposure prophylaxis (PrEP) is an effective HIV prevention tool that requires the ongoing support of physicians to be accessible. Recently, Nova Scotia experienced a $100 \%$ increase in HIV diagnoses. The purpose of this study is to explore the relationship between physicians' support of PrEP, knowledge of PrEP, and PrEP prescribing history using the information-motivation-behavioral (IMB) skills model.

Methods: An online survey was distributed to physicians in Nova Scotia, Canada, and eighty physicians participated. Two exploratory factor analyses were conducted with items from the Support of PrEP scale and Knowledge of PrEP scale. A mediation analysis was conducted to assess if knowledge of PrEP mediated the relationship between support of PrEP and whether physicians have prescribed PrEP in the past.

Results: On average, physicians reported strong support for PrEP, and as support for PrEP increased so did knowledge of PrEP. Further, physicians who had prescribed PrEP demonstrated strong knowledge of PrEP and physicians who had not prescribed PrEP reported feeling neutral. The $95 \%$ bootstrap confidence interval indirect effect of Support for PrEP on prescription history did not include zero $(\mathrm{B}=1.59,95 \%$ BsCI $[0.83,3.57])$ demonstrating that the effect of support for PrEP is mediated by knowledge of PrEP. The most commonly identified barrier to prescribing PrEP was the lack of drug coverage among patients.

Conclusion: The results of the mediation analysis support the IMB skills model regarding support for PrEP, Knowledge of PrEP, and having prescribed PrEP in the past. Our findings suggest that to improve PrEP uptake in Nova Scotia, educational interventions for physicians and universal coverage of the drug would be necessary.

Keywords: HIV pre-exposure prophylaxis, health care providers, attitudes, knowledge, barriers, accessibility, health care access, information-motivation-behavior skills model

\section{Introduction}

Pre-exposure prophylaxis (PrEP) is the use of specific antiretrovirals on a regular basis by people at elevated risk, used for the prevention of HIV. When taken daily, PrEP in the form of co-formulated tenofovir disorproxil fumarate with emtricitabine (or tenofovir alafenamide with emtricitabine) has been demonstrated to be one of the most effective prevention strategies and is particularly noted for drastic reductions in HIV transmission rates among populations at greater risk for HIV. ${ }^{1,2}$ In fact, PrEP has demonstrated a $92-100 \%$ reduction in HIV transmission rate among men who have sex with men (MSM) and transgender women. ${ }^{1,3}$ Despite a growing body of evidence in support of PrEP and the approval of its usage in Canada in 2016, ${ }^{4}$
Correspondence: Jad Sinno School of Health and Human Performance, Dalhousie University, Stairs House, 6230 South Street, Halifax, NS, B3H 4R2, Canada $\mathrm{Tel}+\mathrm{I}(902) 402-9596$

Fax +I (902) 494-5I 20

Email jad.sinno@dal.ca 
many Canadian physicians remain unfamiliar with PrEP medications and its ability to prevent HIV transmission. ${ }^{5}$ The lack of physician knowledge of PrEP poses significant challenges for widespread and effective implementation of this treatment as a biomedical prevention method because PrEP requires the ongoing involvement of health care professionals. $^{6}$

Physicians lie at the forefront of HIV transmission prevention through public outreach, education of patients, and prescription of medications. Previous research into physicians' willingness to prescribe PrEP medications has been shown to be influenced by many factors, including patient demographics, access to the medications, expected adherence, and physician understanding of PrEP efficacy. ${ }^{6,7}$ As such, there is variability in physician perception, familiarity, concerns, and ultimately willingness to prescribe PrEP. Early Canadian surveys of physicians' prescription practices of PrEP found that while there was increasing knowledge of PrEP among doctors, only few have prescribed it. ${ }^{5,8}$ Physicians with greater familiarity of PrEP are more likely to prescribe PrEP than those who were less familiar or without prior knowledge of the drug. ${ }^{5,9,10}$ Similarly, research has shown that greater knowledge of PrEP is correlated with having prescribed PrEP in the past, as well as future intent to prescribe PrEP to patients. $8,10-12$

More recently, a study in 2017 by Walsh and Petroll ${ }^{10}$ used the information-motivation-behaviour (IMB) model to predict PrEP prescription among US primary care physicians. The IMB model describes physician's behaviours based on the information that they have, the motivations and attitudes toward practice, and the behavioural skills that they possess. Several studies have reported that health care providers' behaviours are associated with their knowledge and attitudes, supporting the IMB model. ${ }^{13-15}$ Walsh and Petroll ${ }^{10}$ found that physicians' knowledge of PrEP and attitudes toward PrEP significantly predicted whether physicians had prescribed PrEP in the past. Moreover, greater knowledge of PrEP was associated with greater support for PrEP. Similar to previous findings, a physician's support and knowledge of PrEP both influence a physician's decision to prescribe.

\section{The Current Study}

Despite substantial efforts to curb the spread of HIV in Canada, a 2017 surveillance report identified 2402 new HIV cases across the country. ${ }^{16}$ One region of particular concern is Nova Scotia, which experienced a $100 \%$ increase in HIV diagnoses in 2018 as compared to 2017, with 31 and 15 new cases, respectively. ${ }^{17}$ This increase is unparalleled by any other province or territory and calls for investigating the barriers to effectively implementing HIV PrEP in Nova Scotia.

The purpose of the following study is to investigate the predictors of prescribing PrEP among physicians in Nova Scotia. Informed by the IMB model, this study sought to determine the relationship between physicians' support for PrEP, their knowledge of PrEP, and their history of prescribing PrEP. We hypothesized that:

- Hypothesis (1): Nova Scotia physician's support for PrEP and knowledge of PrEP will be associated with a greater likelihood of having prescribed PrEP in the past, and

- Hypothesis (2): the relationship between support for PrEP and having prescribed PrEP in the past will be mediated by knowledge of PrEP.

Both the proportion of the patient population that is HIV+ and those that at high risk of HIV-acquisition have been included as covariates. These were included to control for the possibility that physician's prescription history was influenced by the proportion of patients that needed PrEP. The covariates also account for the potential that a physician's history or experience treating patients living with HIV may also influence their knowledge or history of prescribing PrEP. Further, this study explored differences among Nova Scotian physicians who had prescribed PrEP and not prescribed PrEP in the past, to better understand the concerns and barriers that are preventing further prescription. Together, these findings aim to inform the development of education and policy changes that are needed for physicians to confidently and competently prescribe PrEP to patients that are at risk for contracting HIV.

\section{Methods}

The questionnaire and methodology for this study was approved by the Human Research Ethics board of Dalhousie University (REB Number: 2018-4481). Informed consent was obtained from all individual participants included in the study.

\section{Participants and Recruitment}

Eligible participants were doctors and nurse practitioners registered and practicing in Nova Scotia, as well as medical residents. A convenience sample was invited to 
participate in the study via email, which described the study and included a link to the survey. The email was circulated to health care providers in Nova Scotia through various health organizations' administrators. The following organizations circulated the email: Doctors Nova Scotia (2361 practicing physicians and 641 residents on their listserv); Halifax Sexual Health Centre; Maritime Resident Doctors; and the Nurse Practitioners Association of Nova Scotia. Subsequent to the initial invite, three follow-up emails were sent to remind and encourage physicians and nurses to complete the survey. Survey responses were anonymous; however, participants could provide an email to be entered into a draw to win a $\$ 100$ gift card. Data collection began in August 2018 and was ongoing until May 2019. The following survey items are pertinent to the analysis in this report.

\section{Survey Instrument}

An online survey was designed on the web-based survey platform, Qualtrics, and focused on the knowledge, opinions, and prescription practices of health care providers regarding PrEP. The survey comprised a total of 52 questions. However, the survey was designed with conditional branching, meaning that questions may or may not be presented based on participants' answers. Participants were also allowed to skip any questions that they did not wish to answer. In addition to collecting practitioner information, the aim of the survey was to assess health care providers' familiarity with PrEP medications, PrEP prescription history and practices, concerns with prescribing PrEP, and the perceived role of PrEP in the future of HIV prevention.

\section{Practitioner Information}

Participants were asked what type of physician they were, the type of setting that they practiced in, and how long they have worked in an independent clinic. Participants were also asked to estimate the proportion of their patient population that is HIV positive and the proportion that is at high risk of HIV-acquisition; these were self-report measures.

\section{Support for PrEP}

Participants were asked 16 questions regarding their opinions of PrEP (Table 1). Questions were adapted from a previous study exploring physicians' attitudes and knowledge of PrEP, with more created for the purposes of the current study. ${ }^{5}$ The questions were measured on a 5-point Likert scale from "Strongly Disagree" to "Strongly Agree". Following a factor analysis (see statistical analyses), nine items were averaged to calculate a score of support for PrEP; greater values indicated greater support for PrEP. Support for PrEP was considered an indication of motivation to prescribe PrEP.

\section{Knowledge of PrEP}

Participants were asked four questions rating their familiarity with PrEP (Table 2). Two questions were measured on a 5-point Likert scale from "Not familiar at all" to "Very Familiar" and "Strongly Disagree" to "Strongly Agree". The other two questions were measured on a 3-point Likert scale from "Not familiar at all" to "Very familiar". The latter two items were adjusted to be on a 5-point Likert scale. Following a factor analysis (see statistical analyses), the four questions were averaged to calculate a score for knowledge of PrEP; greater values indicated greater knowledge of PrEP.

Participants were also asked to report the various media where they heard of PrEP in the past. The possible answers included: peer-reviewed medical journal; HIV/AIDS-related or other medical conference; workshop, lecture or seminar; client/patient website or blog; social media; news media (radio, newspaper or magazine); and other.

\section{History of PrEP Prescription and Barriers}

Participants were asked whether or not they have prescribed PrEP in the past. They were also asked to identify barriers that inhibit them from prescribing PrEP. The possible answers included: I am not familiar enough with PrEP to prescribe it; I am unsure of which patients to prescribe it to; I do not think my patients would be interested in PrEP as an option; my patients are unable to get the drug costs covered; I do not feel there are sufficient data to support its use; there are no barriers that inhibit me from prescribing PrEP; and, other. Lastly, participants were asked what areas of PrEP prescription education is needed to feel more comfortable prescribing PrEP to a high-risk patient. The possible answers included: I do not need any further information; guidelines for prescription; coverage for prescription; side-effects and severity; development of anti-viral resistance; changes in risktaking behavior; monitoring and testing practices; alternative prevention and treatment methods; other.

\section{Statistical Analyses}

All statistical analyses were conducted using SPSS v25. 
Table I Factor Loadings for Opinions of PrEP Scale

\begin{tabular}{|c|c|c|c|}
\hline Item & Question & Factor & $\begin{array}{l}\text { Factor } \\
2\end{array}$ \\
\hline 15 & Investing in PrEP would be an appropriate use of healthcare resources & 0.84 & \\
\hline $16^{R}$ & There is not enough evidence available to justify making PrEP widely available in Canada & 0.73 & \\
\hline 4 & PrEP is an exciting new HIV prevention tool and should be made more widely available as soon as possible & 0.70 & 0.41 \\
\hline 5 & PrEP is a valuable addition to condoms as a prevention option & 0.69 & \\
\hline 2 & PrEP should be covered by the provincial formulary & 0.68 & \\
\hline 12 & PrEP is cost-effective & 0.62 & \\
\hline 6 & $\begin{array}{l}\text { Physicians have an ethical obligation to make available any intervention that could decrease an individual's risk of } \\
\text { becoming infected with HIV }\end{array}$ & 0.58 & \\
\hline 13 & $\begin{array}{l}\text { I am concerned about unequal access for certain groups if funding for PrEP medications is out-of-pocket or through } \\
\text { private insurance }\end{array}$ & 0.55 & \\
\hline$I^{R}$ & PrEP is dangerous and should not be prescribed & 0.49 & \\
\hline $7^{R}$ & I worry about the risk for development of antiviral drug resistance if a person using PrEP becomes infected & & 0.71 \\
\hline $10^{R}$ & I worry that patients may not adhere to necessary monitoring and testing while taking PrEP & & 0.70 \\
\hline$I^{R}$ & I worry that patients may not take PrEP medications as directed, thus reducing its efficacy & & 0.60 \\
\hline $14^{R}$ & $\begin{array}{l}\text { PrEP could lead to the "medicalization" of HIV prevention and take focus away from other, more important } \\
\text { prevention efforts }\end{array}$ & & 0.58 \\
\hline $8^{R}$ & I worry about potential side effects and their severity & & 0.50 \\
\hline $9^{R}$ & $\begin{array}{l}\text { I worry that PrEP use may increase risk taking (behavioural disinhibition / risk compensation: increased risk-taking } \\
\text { behavior due to increased sense of protection) }\end{array}$ & & 0.48 \\
\hline $3^{R}$ & PrEP has the potential to do more harm than good if not carefully implemented & 0.45 & 0.48 \\
\hline
\end{tabular}

Notes: Factor analysis of 16 items measuring physicians' opinions of PrEP. Principal Axis factoring extraction method, with Varimax (orthogonal) rotation. The Kaiser-MeyerOlkin (KMO) measure of sampling adequacy was 0.84 . Bartlett's test of sphericity was significant $\left(\chi^{2}(I 20)=590.77, p<0.05\right)$. Participants were asked to what extent they agreed with the above statements on a 5-point Likert scale from "Strongly disagree" to "Strongly agree". This two-factor structure was verified using scree plot and parallel analysis. The first factor was identified as "support for PrEP" and the second factor was "concern for PrEP". Items 3 and 4 cross-loaded, but were assigned to the factor with the strongest loading. Cronbach's $\alpha=0.88$.

${ }^{R}$ Items were reversed prior to conducting factor analysis.

\section{Factor Analysis}

Two exploratory factor analyses were conducted with the items from the Support of PrEP Scale and Knowledge of PrEP scale. Nine items from the Support of PrEP Scale were reverse scored prior to analysis (Table 1). The Kaiser-MyerOlkin (KMO) measure of sampling adequacy was checked to ensure a sufficient sample size to conduct the factor analyses; a KMO score greater than 0.5 was considered sufficient. ${ }^{18}$ The Bartlett test for sphericity was used to assess whether the correlation matrix of the items were significantly different than an identity matrix; if the test was significant at $\mathrm{p}<0.05$, the data were considered adequate to conduct a factor analysis. ${ }^{19}$ Principal axis factoring was the extraction method used. The number of factors extracted were determined through a parallel analysis, which was conducted using the $\mathrm{O}^{\prime}$ Connor $^{20}$ SPSS Macro. If more than one factor was extracted, varimax rotation was applied. Items that crossloaded on multiple factors were associated as part of the factor that they most strongly loaded with. The reliability of the newly calculated variables was measured by Cronbach's alpha; values above 0.70 denote good internal consistency. ${ }^{21}$

\section{Mediation}

A mediation analysis was conducted using PROCESS macro v3.3 in SPSS v25, to assess if Support for PrEP predicted whether or not physicians have prescribed PrEP in the past (dichotomous outcome variable). The mediator variable was Knowledge of PrEP, and two covariates were 
Table 2 Factor Loadings for Knowledge of PrEP Scale

\begin{tabular}{|l|l|l|}
\hline Item & Question & Factor \\
\hline I & Please rate your level of familiarity with the following evidence-based HIV prevention options: Pre-exposure prophylaxis (PrEP) & 0.99 \\
$2^{\text {a }}$ & How would you describe your current knowledge about PrEP? & 0.86 \\
4 & I have enough current knowledge about PrEP to make informed prescribing decisions & 0.85 \\
$3^{\text {a }}$ & $\begin{array}{l}\text { How familiar are you with the Canadian guideline on HIV pre-exposure prophylaxis and non-occupational post-exposure } \\
\text { prophylaxis? }\end{array}$ & 0.72 \\
\hline
\end{tabular}

Notes: Factor analysis of four items measuring knowledge of PrEP. Principal Axis factoring extraction method, with no rotation because only one factor was extracted. The Kaiser-Meyer-Olkin (KMO) measure of sampling adequacy was 0.80 . Bartlett's test of sphericity was significant $\left(\chi^{2}(6)=240.18, p<0.05\right)$. This single factor structure was verified based on eigen value, scree plot, and parallel analysis. Item I was measured on a 5-point Likert scale from "Not familiar at all" to "Very familiar". Item 4 was measured on a 5-point Likert scale from "Strongly disagree" to "Strongly agree". Cronbach's $\alpha=0.91$. "Items were measures on a Likert scale from I "Not familiar at all" to 3 "Very familiar", but were modified to be on a scale from I to 5.

included in the analysis: proportion of patient population that was HIV+; and the proportion of the population that was at risk of HIV-acquisition. PROCESS model 4 was used with 5000 bootstrap samples to calculate the $95 \%$ confidence intervals. A heteroscedasticity consistent standard error and covariance matrix estimator, $\mathrm{HC} 4,{ }^{22}$ was used to adjust for the possibility of unequal variance in the data. The first path in the mediation analysis was a multiple linear regression predicting Knowledge of PrEP. The assumptions of the linear regression were assessed prior to conducting the analysis. Normally distributed residuals were confirmed using residual plots in SPSS. The Durbin-Watson statistic was used to check for independent errors; a value greater than 1.5 was sufficient. The variance inflation factors were checked to ensure the absence of multicollinearity; values less than 10 were considered sufficient. $^{23}$ The second step in the mediation analysis was a binary logistic linear regression. The assumptions of the binary logistic regression were also assessed prior to conducting the mediation analysis. The linearity of the logit assumption for all variables was confirmed using the Box-Tidwell approach. ${ }^{18}$ The independence of errors assumptions was presumed due to study design; physicians were invited to participate in the survey anonymously and independently of one another. Outliers were identified by saving Leverage values, and the mediation analysis was conducted with and without outliers (observations with leverage values greater than 3 $(\mathrm{k} / \mathrm{N}))$ to ensure that the interpretation of the results did not change. ${ }^{18,24}$ The total effect model is not available in mediation with a dichotomous outcome variable; however, the presence of mediation can be assessed by evaluating the $95 \%$ bootstrap confidence intervals (BsCI) of the indirect effect. $^{25}$

\section{Results \\ Participants}

The online survey was accessed by 110 participants and completed by 80 . No nurses or medical residents responded to the survey, and as such, the sample was comprised entirely of physicians. Given the convenience-sampling strategy of circulating emails to several listservs, a response rate could not be calculated; particularly because we could not confirm that potential participants may be subscribed to multiple listservs. On average, it took participants 14.72 minutes to complete the survey. Of those who completed the survey, only 25 (31.3\%) had prescribed PrEP in the past. Table 3 summarizes the descriptive statistics by physician's prescribing history. A majority of participants $(n=50,62.50 \%)$ were general practitioners, 22 of whom had prescribed PrEP in the past $(44.00 \%)$ and $28(56.00 \%)$ had not. Several participants $(\mathrm{n}=$ $23,28.75 \%$ ) selected "other" as their specialty, they were most commonly emergency physicians $(n=6,7.50 \%)$, but also included addiction specialists $(\mathrm{n}=2,2.50 \%)$, anesthesiologists $(\mathrm{n}=2,2.50 \%)$, neonatologists $(\mathrm{n}=2,2.50 \%)$, psychiatrists $(\mathrm{n}=$ 2, 2.50\%), urologists $(n=2,2.50 \%)$, cardiologists $(n=1$, $1.25 \%)$, endocrinologists $(\mathrm{n}=1,1.25 \%)$, hematologists $(\mathrm{n}=$ $1,1.25 \%)$, medical microbiologists $(\mathrm{n}=1,1.25 \%)$, paediatric oncologists $(n=1,1.25 \%)$, and rheumatologists $(n=1,1.25 \%)$; one participant did not specify their speciality. This sample of physicians in Nova Scotia most commonly practiced in a private setting $(\mathrm{n}=29,36.25 \%)$, with roughly equal proportions having prescribed PrEP $(\mathrm{n}=10,40.00 \%)$ and having never prescribed PrEP ( $\mathrm{n}=19,34.55 \%)$. On average, physicians who had prescribed PrEP in the past spent 17.40 years (se $=2.18$ ) practicing post-residency, as compared to 14.92 years ( $\mathrm{se}=1.97$ ) among physicians who have not prescribed PrEP. Nevertheless, there were roughly equal percentages of the patient population who were HIV+ among physicians who 
Table 3 Descriptive Statistics

\begin{tabular}{|c|c|c|c|}
\hline \multirow[t]{2}{*}{ Variables } & Total & Prescribed PrEP & Not Prescribed PrEP \\
\hline & \multicolumn{3}{|l|}{$\mathbf{N}(\%)$} \\
\hline Total & $80(100)$ & $25(100)$ & $55(100)$ \\
\hline \multicolumn{4}{|l|}{ Physician Type } \\
\hline General practitioner & $50(62.50)$ & $22(88.00)$ & $28(50.91)$ \\
\hline Infectious diseases specialist & $5(6.25)$ & $2(8.00)$ & $3(5.45)$ \\
\hline General internist & $2(2.50)$ & - & $2(3.64)$ \\
\hline Other & $23(28.75)$ & I (4.00) & $22(40.00)$ \\
\hline \multicolumn{4}{|l|}{ Practice Setting } \\
\hline Private practice & $29(36.25)$ & $10(40.00)$ & $19(34.55)$ \\
\hline Community hospital & $8(10.00)$ & I (4.00) & $7(12.73)$ \\
\hline Academic hospital & $21(26.25)$ & $2(8.00)$ & $19(34.55)$ \\
\hline Community health center & $12(15.00)$ & $4(16.00)$ & $8(14.55)$ \\
\hline Walk-in clinic & $\mathrm{I}(1.25)$ & I (4.00) & - \\
\hline Sexual health clinic & $3(10.00)$ & $3(12.00)$ & - \\
\hline \multirow[t]{2}{*}{ Other } & $6(7.50)$ & $4(16.00)$ & $2(3.64)$ \\
\hline & \multicolumn{3}{|l|}{ Mean (SE) } \\
\hline Years of practice post-residency & $15.69(1.5 \mathrm{I})$ & $17.40(2.18)$ & $14.92(1.97)$ \\
\hline \% Patient Population that is HIV+ & $\mathrm{I} .40(0.3 \mathrm{I})$ & $1.83(0.89)$ & $1.21(0.23)$ \\
\hline \% Patient population at risk of $\mathrm{HIV}$-acquisition & $8.93(1.71)$ & $9.0(2.18)$ & $8.9(2.26)$ \\
\hline Support for PrEP & $3.99(0.06)$ & $4.24(0.09)$ & $3.87(0.08)$ \\
\hline Knowledge of PrEP & $3.08(0.13)$ & $4.16(0.13)$ & $2.59(0.14)$ \\
\hline
\end{tabular}

have $($ mean $=1.83 \%$, se $=0.89)$ and have not prescribed PrEP (mean $=1.21 \%$, se $=0.23$ ). There was, also, a relatively equal proportion of the patient population that was at high risk of HIV-acquisition among physicians who had (mean $=9.0 \%$, se $=2.18)$ and had not prescribed $\operatorname{PrEP}($ mean $=8.9 \%$, se $=2.26)$.

\section{Support for PrEP}

The results of the parallel analysis revealed a two-factor structure for the 16 items asking physicians' opinions of PrEP. Nine items loaded on the first factor, which was identified as Support for PrEP (See Table 1 for factor loadings). The remaining items loaded on a factor that was identified as being Concern for PrEP. This two-factor structure was confirmed using verified using scree plot and parallel analysis, which suggests that support and concern for PrEP are separate variables; ${ }^{18}$ only Support for PrEP was used in subsequent analyses. The KMO statistic was 0.84 and the Bartlett's test of sphericity was significant $\left(\chi^{2}\right.$ $(120)=590.77, \mathrm{p}<0.05)$, satisfying the assumptions of factor analysis. The Cronbach's $\alpha$ was 0.88 , demonstrating good internal consistency. In general, both physicians who have prescribed PrEP in the past $($ mean $=4.24$, se $=0.09$ ) and those who have not (mean $=3.87$, se $=0.08$ ) show strong support for PrEP (Table 1).

\section{Knowledge of PrEP}

The results of the parallel analysis revealed a one-factor structure for the four items asking physicians their knowledge of PrEP (See Table 2 for factor loadings). The KMO statistic was 0.80 and the Bartlett's test of sphericity was significant $\left(\chi^{2}(6)=240.18, p<0.05\right)$, satisfying the assumptions of factor analysis. The Cronbach's $\alpha$ was 0.91 , demonstrating good internal consistency. On average, the Nova Scotian physicians in our sample who have prescribed PrEP demonstrated strong knowledge of PrEP (mean $=4.16$, se $=0.13$ ), whereas physicians who have not prescribed PrEP reported feeling neutral (mean $=2.59$, se $=0.14$ ) about their knowledge of PrEP (Table 2).

\section{Mediation Analysis}

Table 4 reports the bivariate Pearson correlations between all continuous variables. There was a significant positive relationship between Support for PrEP and both Knowledge of PrEP $(r=0.46, p<0.01)$ as well as the 
Table 4 Bivariate (Pearson) Correlations

\begin{tabular}{|c|c|c|c|}
\hline & $\begin{array}{l}\text { Knowledge of } \\
\text { PrEP }\end{array}$ & $\begin{array}{l}\text { Support for } \\
\text { PrEP }\end{array}$ & $\begin{array}{l}\text { \% PP } \\
\text { HIV+ }\end{array}$ \\
\hline Support for PrEP & $0.46 * *$ & & \\
\hline \% PP HIV+ & $0.22 *$ & 0.16 & \\
\hline $\begin{array}{l}\text { \% PP at risk of HIV- } \\
\text { acquisition }\end{array}$ & 0.18 & $0.33^{* *}$ & 0.40 ** \\
\hline
\end{tabular}

Notes: $*_{p}<0.05, *^{*} p<0.01$ two-tailed.

Abbreviation: PP, patient population.

percentage of the patient population that is at risk for HIVacquisition $(\mathrm{r}=0.33, \mathrm{p}<0.01)$. There was also a positive relationship between the percentage of the patient population that is HIV+ and both Knowledge of PrEP ( $r=0.22$, $\mathrm{p}<0.05$ ) as well as the percentage of the patient population that is at risk for HIV-acquisition $(\mathrm{r}=0.40, \mathrm{p}<0.01)$.

See Figure 1 for a model representing the mediation analysis of predictors of PrEP Prescription history. There is a significant positive relationship between Support for PrEP and Knowledge of PrEP $(\mathrm{B}=0.91, \mathrm{p}<0.05,95 \%$ BsCI [0.48, 1.33]; Table 5), while controlling for both the percentage of the patient population that is HIV+ and at risk of HIV-acquisition. That is to say that physicians with greater support of PrEP also had a greater knowledge of PrEP. Both the percentage of the patient population that is $\mathrm{HIV}+(\mathrm{B}=0.07, \mathrm{p}=0.09,95 \% \mathrm{BsCI}[-0.01,0.15])$ and at risk of HIV-acquisition $(\mathrm{B}=0.00, \mathrm{p}=0.95,95 \% \mathrm{BsCI}$ $\left[\begin{array}{ll}-0.05,0.05\end{array}\right]$ were nonsignificant predictors of Knowledge of PrEP (Table 5). The variables in the regression analysis predicting Knowledge of PrEP accounted for $23 \%$ of the variance explained $\left(\mathrm{R}^{2}\right)$.

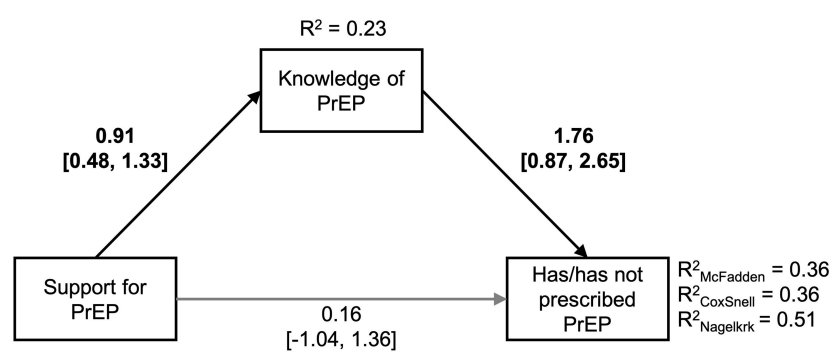

Figure I Model representing Support for PrEP predicting physicians' history of having prescribed PrEP, as mediated through Knowledge of PrEP. Covariates included in the model, but not in the diagram, are proportion of patient population that are $\mathrm{HIV}+$ and proportion of patient population at high risk of HIV acquisition. Total effect model is not available for dichotomous outcome variable (has/has not prescribed PrEP). Diagram reports unstandardized beta for each path and the bootstrap $95 \%$ confidence interval within parentheses. 5000 bootstrap samples were used for the confidence intervals. The indirect effect of Support for PrEP on has/has not prescribed PrEP is B $=1.59,95 \%$ $\mathrm{BsCl}[0.83,3.57]$, demonstrating that mediation has taken place. Bolded black lines represent significant paths at $p<0.05$. (see Table 5 for linear regression results and Table 6 for binary logistic regression results).
Table 5 Linear Regression Model of Predictors of Knowledge of PrEP

\begin{tabular}{|l|l|l|l|l|}
\hline & $\mathbf{B}$ & $\mathbf{S E}^{\mathbf{a}}$ & $\boldsymbol{\beta}$ & $\mathbf{P}$ \\
\hline Support for PrEP & $\mathbf{0 . 9 1}[\mathbf{0 . 4 8}, \mathbf{1 . 3 3}]$ & $\mathbf{0 . 2 1}$ & $\mathbf{0 . 4 4}$ & $\mathbf{0 . 0 0}$ \\
HIV-positive & $0.07[-0.01,0.15]$ & 0.04 & 0.16 & 0.09 \\
Risk of HIV-acquisition & $0.00[-0.05,0.05]$ & 0.03 & -0.21 & 0.95 \\
Constant & $-0.64[-2.36,1.07]$ & 0.86 & & 0.46 \\
\hline
\end{tabular}

Notes: Multiple linear regression results of predictors of knowledge of PrEP. 95\% Bootstrap Confidence interval of $B$ are listed between parentheses. Bolded variables are significant at $p<0.05$. Total variance explained by the model is $R^{2}=0.23 . N=78$. ${ }^{a}$ Heteroscedasticity-consistent SE estimators, adjusted using PROCESS HC4. ${ }^{22}$

Abbreviations: $\mathrm{B}$, unstandardized beta; SE, standard error; $\beta$, standardized beta.

Physicians' Knowledge of PrEP was the only significant predictor of PrEP prescription history $(\mathrm{B}=1.76, \mathrm{p}<$ $0.05,95 \%$ BsCI $[0.87,2.65]$; OR $=5.81,95 \%$ CI $[2.39$, 14.16]; Table 6); this was true while controlling for both the percentage of the patient population that is $\mathrm{HIV}+$ and at risk of HIV-acquisition. As physicians' knowledge of PrEP increased, so did the likelihood that they have prescribed PrEP in the past. The two covariates, percent of patient population that is $\mathrm{HIV}+(\mathrm{OR}=1.00,95 \% \mathrm{CI}[0.82$, 1.23]), and percent of population that is at risk of HIVacquisition $(\mathrm{OR}=0.96,95 \% \mathrm{CI}[0.91,1.02])$ were both non-significant predictors of prescription history. The variables in the logistic regression predicting prescription history had the following pseudo- $\mathrm{R}^{2}$ values: McFadden $=$ 0.36 , CoxSnell $=0.36$, Nagelkerke $=0.51$.

The direct effect of Support for PrEP on prescription history was non-significant $(\mathrm{OR}=1.18,95 \% \mathrm{CI}[0.35$, $3.91]$ ). Furthermore, the $95 \%$ bootstrap confidence interval indirect effect of Support for PrEP on prescription history does not include zero ( $\mathrm{B}=1.59,95 \%$ BsCI $[0.83,3.57])$, indicating that the effect of Support of PrEP is mediated by knowledge of PrEP. That is, as support for PrEP increases, knowledge of PrEP also increases, which in turn, results in a greater likelihood that PrEP would have been prescribed in the past by a physician.

\section{Barriers to Prescribing PrEP}

Of the physicians in our sample who did not prescribe PrEP in the past, nearly one-fifth $(\mathrm{n}=10,18 \%)$ have not heard of PrEP (Figure 2). Yet, a majority of physicians heard of PrEP from peer-reviewed medical journals among both physicians who have prescribed PrEP ( $\mathrm{n}=17,68 \%$ ) and who have not $(\mathrm{n}=28,51 \%)$; this was the most common avenue for having heard of PrEP (Figure 2). A lack of physician knowledge of PrEP was identified as 
Table 6 Binary Logistic Regression Model of Predictors of Having Prescribed PrEP in the Past

\begin{tabular}{|l|l|l|}
\hline & B & OR \\
\hline Support for PrEP & $0.16[-1.04,1.36]$ & $1.18[0.35,3.91]$ \\
Knowledge of PrEP & $\mathbf{1 . 7 6}[\mathbf{0 . 8 7}, \mathbf{2 . 6 5}]$ & $\mathbf{5 . 8 I}[\mathbf{2 . 3 9}, \mathbf{1 4 . 1 6 ]}$ \\
HIV-positive & $0.00[-0.20,0.21]$ & $1.00[0.82,1.23]$ \\
Risk of HIV-acquisition & $-0.04[-0.09,0.02]$ & $0.96[0.91,1.02]$ \\
Constant & $-7.22[-12.10,-2.53\}$ & 0.00 \\
\hline
\end{tabular}

Notes: Binary logistic regression results of predictors of having prescribed PrEP in the past. 95\% Bootstrap Confidence Intervals for B and OR are listed in parentheses. Bolded variables are significant at $\mathrm{p}<0.05$. Total variance explained by the model is Pseudo- $\mathrm{R}^{2}:$ McFadden $=0.36$, CoxSnell $=0.36, \mathrm{Nagelkerke}=0.5 \mathrm{I} . \mathrm{N}=78$.

Abbreviations: $B$, unstandardized beta; OR, odds ratio.

one of the most common barriers to prescribing it to patients among physicians who have not prescribed PrEP in the past $(\mathrm{n}=32,58 \%$; Figure 3$)$. However, the most commonly identified barrier by both physicians who have $(\mathrm{n}=18,72 \%)$ and have not $(\mathrm{n}=29,53 \%)$ prescribed PrEP in the past was the lack of drug coverage among their patients (Figure 3).

When asked what areas of PrEP prescription education would be needed to prescribe PrEP in the future, a substantial portion of physicians who have prescribed PrEP in the past said that they do not need any further information $(\mathrm{n}=10,40 \%$; Figure 4$)$. However, similar proportions reported needing further information pertaining to: coverage for prescription $(\mathrm{n}=12,48 \%)$; monitoring and testing practices $(\mathrm{n}=11,44 \%)$; guidelines for prescription $(\mathrm{n}=9,36 \%)$; side-effects and severity $(\mathrm{n}=$ 9, 36\%); as well as development of anti-viral resistance $(\mathrm{n}=5,20 \%)$. Only $2(4 \%)$ physicians who have not prescribed PrEP in the past reported not needing any further information to prescribe PrEP in the future (Figure 4). The proportion of physicians who did not prescribe PrEP in the past who also reported needing further information regarding $\mathrm{PrEP}$ in the following areas (Figure 4): guidelines for prescription $(n=43$, $78 \%)$; coverage for prescription $(\mathrm{n}=41,75 \%)$; sideeffects and severity $(\mathrm{n}=40,73 \%)$; monitoring and testing practices $(n=39,71 \%)$; development of anti-viral resistance $(n=39,71 \%)$. A smaller portion of doctors who have not prescribed PrEP in the past needed further information pertaining to alternative prevention and

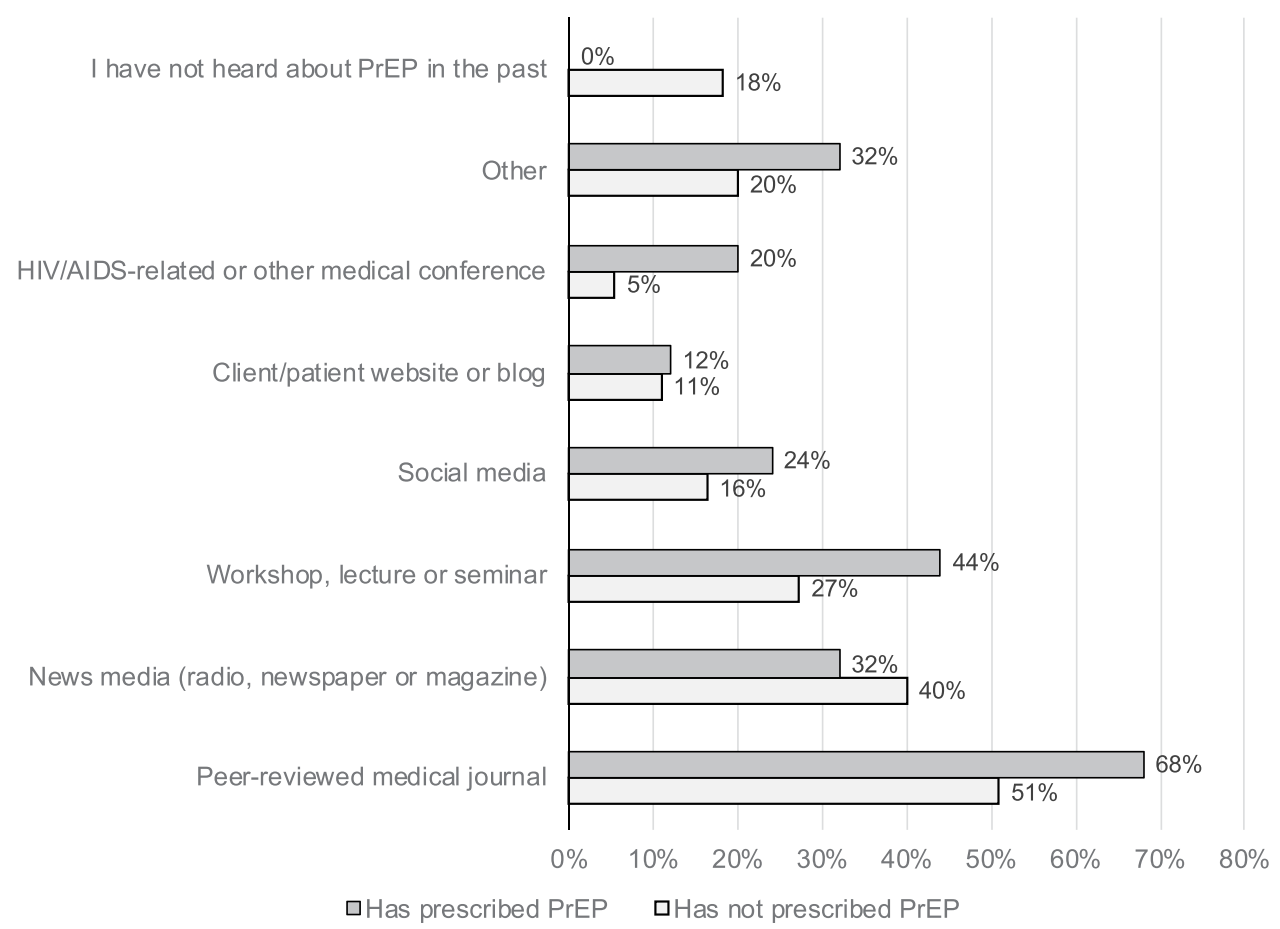

Figure 2 The proportion of physicians who have $(n=25)$ and have not prescribed PrEP $(n=55)$ reporting on the various mediums where they have heard of PrEP in the past. Participants were able to select more than one answer. 


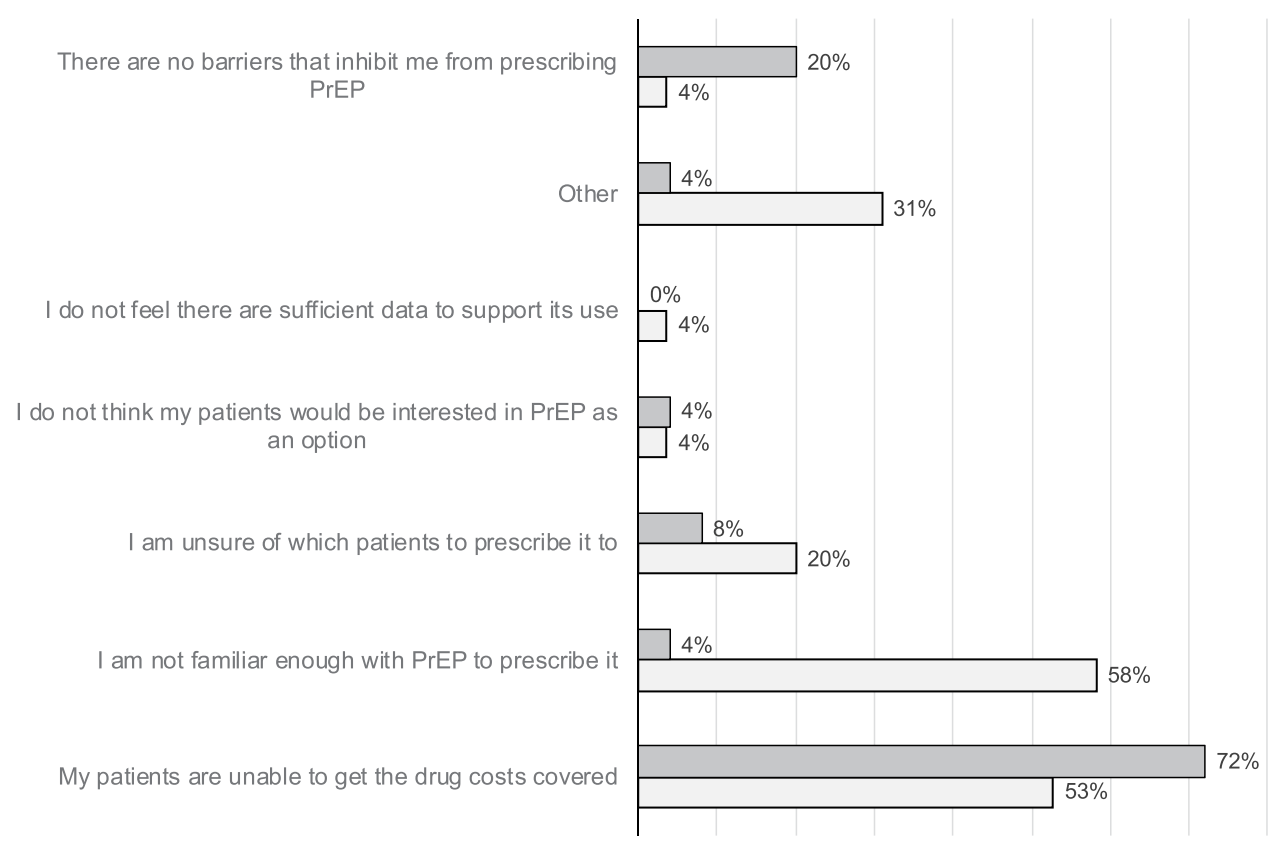

口Has prescribed PrEP $\quad$ 口Has not prescribed PrEP

Figure 3 The proportion of physicians who have $(n=25)$ and have not prescribed $\operatorname{PrEP}(n=55)$ reporting on the various barriers currently inhibiting them from prescribing PrEP. Participants were able to select more than one answer.

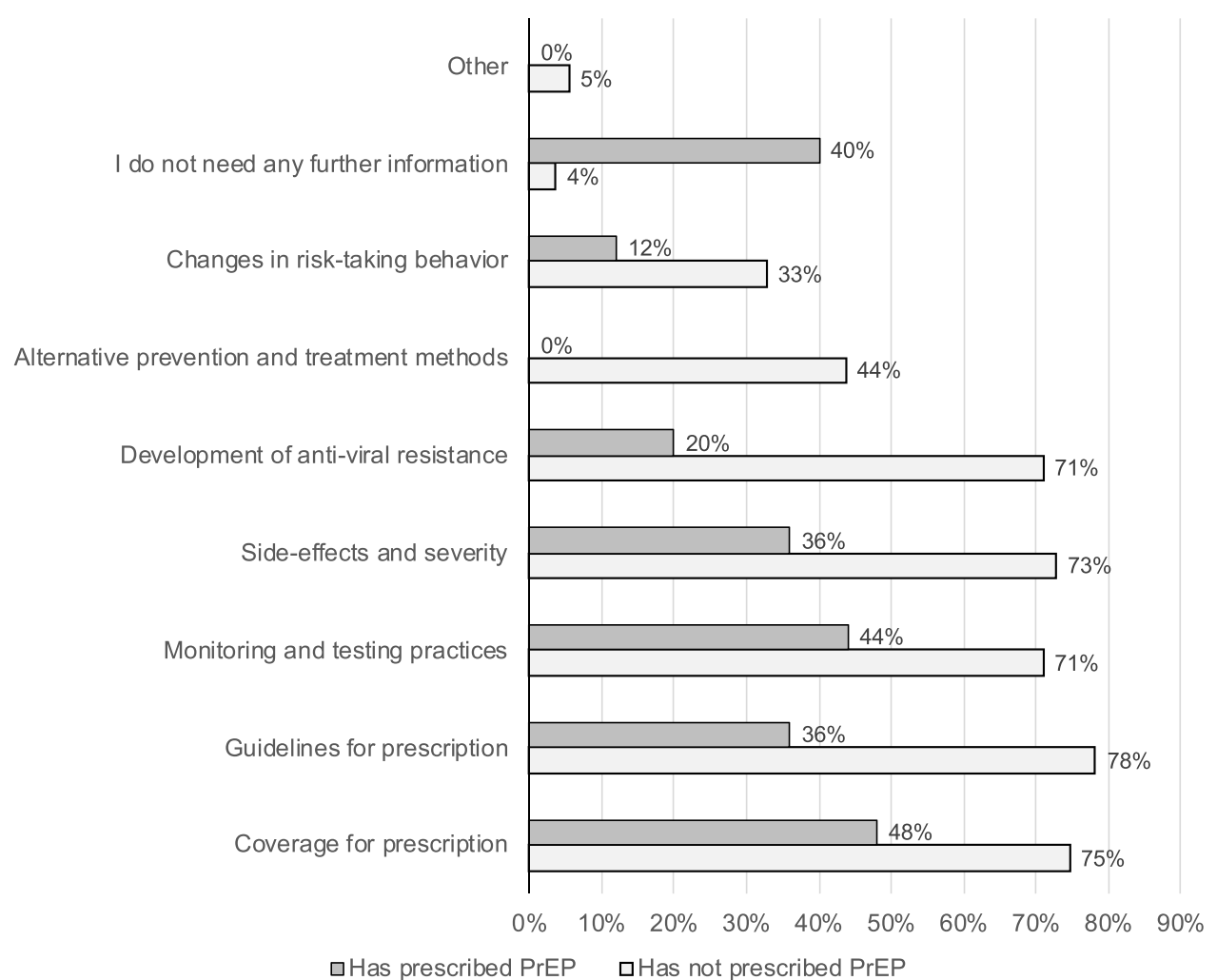

Figure 4 The proportion of physicians who have $(n=25)$ and have not prescribed PrEP $(n=55)$ reporting on areas of PrEP prescription education needed to feel more comfortable to prescribe PrEP to a high-risk patient? Participants were able to select more than one answer. 
treatment methods $(\mathrm{n}=24,44 \%)$ as well as changes in risk-taking behavior $(\mathrm{n}=18,33 \%)$.

\section{Discussion}

Despite the addition of PrEP to the list of approved drugs by Health Canada in 2016 and the publication of the Canadian guidelines regarding prescription in $2017,4,26$ our study has revealed that a similar proportion of Nova Scotian physicians have not heard of PrEP as compared to a 2014 national study. ${ }^{5}$ Of the physicians who were aware of PrEP, which comprised a majority of our sample (87.5\%), only one-third had ever prescribed PrEP. These findings are a substantial increase from 2014, which report that only $12.5 \%$ of physicians had prescribed PrEP in the past. $^{5}$ Among general practitioners in our sample (primary care physicians), less than one-half had prescribed PrEP in the past. These findings are consistent with previous literature from the United States, which demonstrates that only a small fraction of general practitioners sampled had prescribed PrEP. ${ }^{27,28}$ Importantly, our sample revealed that there were similar proportions of patients who were at high risk of HIV-acquisition among physicians who have and have not prescribed PrEP. In turn, it is conceivable that there are patients who could benefit from PrEP prescriptions but are served by physicians who choose to not prescribe it.

Unlike other HIV-prevention methods, such as condom use, $\operatorname{PrEP}$ is a biomedical intervention that requires ongoing participation and monitoring by a medical provider. This means that patients' access to PrEP is contingent on medical providers having the knowledge and willingness to prescribe PrEP. Moreover, in Canada, marginalized communities such as MSM and Indigenous persons make up the majority of new HIV cases. ${ }^{16}$ These communities who continue to be underserved by the health care system are the very communities in most need of access to PrEP. One study from the United States found that marginalized populations, particularly MSM, benefit the most from PrEP interventions. ${ }^{2}$ Several experts have recognized that the end of the HIV epidemic would need to include prescription of PrEP by a wider range of health care providers. ${ }^{6,29-32}$

It is of particular concern that since the release of the Canadian clinical guidelines, ${ }^{4,26}$ many physicians in our sample reported not having enough information to make informed prescribing decisions. A 2018 study by Patel and colleagues in the Saint Louis, Missouri, found that $83 \%$ of patients who sought PrEP were referred to a different general practitioner who would feel more comfortable discussing PrEP. ${ }^{33}$ Nova Scotia suffers from a shortage of primary care physicians, with nearly 50,000 persons waiting to be placed with a family doctor and nurse practitioner as of January 2020. ${ }^{34-36}$ Patients turned away by their general practitioners in Nova Scotia would have tremendous difficulty finding a physician able to provide ongoing care regarding PrEP; this is especially true since STI clinics and walk-in clinics are set up for urgent care, rather than ongoing treatment.

Having the knowledge and skills necessary to prescribe PrEP have been identified as the most critical predictors of willingness to prescribe among health care providers. ${ }^{10,37}$ Our study revealed that, on average, all participants were in support for PrEP; however, only those who have prescribed it in the past felt confident in their knowledge of PrEP. This finding confirms our first hypothesis, that greater knowledge of PrEP would be associated with having prescribed PrEP in the past. Moreover, our analyses revealed that greater support for PrEP led to greater knowledge of PrEP, which ultimately was associated with having prescribed PrEP in the past; this finding confirms our second hypothesis. We suggest that increased support for PrEP is an indication of motivation to prescribe PrEP, and as such, these results support the information-motivational-behavioural skills model as previously demonstrated by Walsh and Petroll. ${ }^{10}$ Our results also suggest that greater knowledge of PrEP would lead to a greater willingness to prescribe PrEP, regardless of the proportion of the patient population that is $\mathrm{HIV}+$ or at high risk of HIV-acquisition. In turn, there are two main avenues through which the medical community can support the implementation of PrEP: 1) promoting physician support of PrEP through the introduction of this medication as an option for prevention alongside more traditional methods; and 2) by increasing access to resources regarding the efficacy and clinical guidelines of HIV PrEP for all physicians.

While a majority of participants reported having heard of PrEP through peer-reviewed medical journals, only onethird had learned about PrEP through a workshop, lecture, or seminar. Novel and interactive educational interventions may be particularly useful in improving health care provider's support and knowledge of PrEP. An increasing number of general practitioners are open to learning how to prescribe PrEP. $^{38}$ A recent study demonstrated that a 1-hour educational session proved effective at improving PrEP practices among medical residents. ${ }^{39}$ Following the 
training, medical residents were more likely to rate PrEP as effective and safe, and reported overall comfort in prescribing PrEP. Moreover, the training session improved medical residents' understanding of PrEP safety, effectiveness, and usefulness. After 6 months, the self-rated likelihood of prescribing PrEP increased from 33\% to $67 \%$.

Further, a simple optimization intervention to support health care providers prescribing PrEP was recently piloted by Saberi and colleagues. ${ }^{40}$ The pilot study revealed that integrating such an intervention would be feasible in clinical settings. Given our findings that physicians did not know which patients to prescribe PrEP to, a similar intervention, which includes a comprehensive HIV-risk assessment tool, would prove useful. Moreover, an ongoing monitoring feature and educational resources would align closely with the educational needs that were identified by our study participants. Specifically, further information pertaining to clinical guidelines, monitoring and testing practices, as well as the side-effects and severity of the drug. In Nova Scotia, an intervention may be particularly helpful for physicians who lack the knowledge of PrEP and would aid in the provision of healthcare for patients who would benefit from this ongoing care.

While educational interventions could help overcome the lack of provider knowledge for the provision of PrEP care, the most commonly identified barrier to prescribing PrEP in our sample was cost. Patient inability to afford the medication has been identified as a significant barrier to access by several sources from the United States. ${ }^{11,30,41-43}$ Many physicians are apprehensive of prescribing PrEP to patients who may not be able to afford it; training and education to improve knowledge of PrEP will not overcome this barrier. Further, a substantial patient-level barrier that prevents highrisk patients from accessing PrEP is low self-perceived HIV risk. ${ }^{44}$ Patients who do not feel that they benefit greatly from PrEP will be especially deterred from adhering to the regimen or paying for it. It is particularly concerning that HIV disproportionally affects MSM and Indigenous people, who, on average, have less wealth than heterosexual and EuroCanadian counterparts, respectively. ${ }^{45}$ In turn, the very populations that would benefit the most from PrEP are also the individuals least likely to be able to afford it.

Currently, Nova Scotia does not offer universal access for PrEP. Patients in Nova Scotia can access PrEP in three ways: 1) pay for the treatment out of pocket, and the cheapest non-name brand is $\$ 255 /$ month; ${ }^{46}$ 2) pay for the treatment with private insurance, but different policies cover only a portion of the cost or not at all; and 3) access coverage through the Nova Scotia Pharmacare program, which does not provide coverage for most patients who need PrEP. ${ }^{47}$ For Nova Scotia to see a significant increase in PrEP uptake and a reduction of new HIV cases, universal coverage for PrEP would have to be introduced in addition to increased health care provider training. Other provinces in Canada have seen success in this regard. For example, following the implementation of universal coverage for PrEP, British Columbia reported a significant increase in its uptake and is on track to end the HIV epidemic in their region. $^{48,49}$

\section{Strengths and Limitations}

This study was the first of its kind to explore the attitudes and practices of physicians across Nova Scotia regarding PrEP. The study design allowed us to invite a wide range of doctors, nurses, and medical residents in Nova Scotia to complete the study. The dissemination of the study via email listservs and online meant that we could reach a broader population. Moreover, without setting any inclusion/exclusion criteria (other than being a physician, nurse, or medical resident), our sampling strategy would have invited health care providers regardless of their preconceptions or biases of PrEP. Through the use of various questionnaires, we were able to better understand the current state of PrEP beliefs and practices among health care practitioners. What remains to be addressed is how HIV PrEP distribution will be handled systematically. Future research that explores optimal distribution methods in Nova Scotia, adequate training opportunities for various professions, and political efforts to ensure that access to PrEP is not hindered by cost.

A notable limitation of this study was the small response rate. Despite circulating the survey to all doctors, nurses, and medical residents in Nova Scotia (estimated to be in excess of 3000 individuals), our survey was only accessed by 110 and only completed by 80 physicians. Our small convenience sample means that our results may not reflect the overall practices and attitudes of physicians in Nova Scotia. However, the relationships that we uncovered are consistent with, and reinforced by, previous literature with larger and more representative samples. A report published in 2018 has outlined similar struggles recruiting health care providers in New York to answer internet surveys regarding their attitudes on PrEP. ${ }^{50}$ Silverman and colleagues ${ }^{50}$ outlined some strategies that seemed to incite greater response from health care providers. Emails using listservs were deemed effective but 
mailed letters would help bolster this recruitment strategy. For our study, introducing a paper-and-pencil survey option may have improved our sample size. The authors also found that reminders did not necessarily encourage response from participants; however, studies that set 2 to 3 reminders at 1 -week intervals seemed to be most successful. It may have been beneficial for us to shorten the recruitment period and send multiple reminders at shorter intervals than we did. Lastly, while incentives were not necessarily effective, they were observed to be more effective than a lottery. In addition to recruiting practitioners who were familiar with PrEP, we aimed to recruit practitioners who were not; in turn, these practitioners may feel that a study regarding PrEP is not relevant to their practice and have opted not to respond. Ultimately, our sample may not represent a wider range of physicians who chose not to engage with our study due to their lack of PrEP awareness and the subsequent perceived unimportance of our research.

The analyses controlled for the physician's proportion of the population that is at risk for HIV and the proportion that is currently living with HIV. These variables are self-report measures and, as such, may result in a form of circular logic. That is, physicians who think that they serve more patients are at higher risk of HIV may also be more likely to prescribe PrEP. Additionally, risk may be perceived differently by different physicians; the same behaviour may be deemed risky by one physician and not another. Future studies ought to use a standard definition for risk and an objective measure of the population that is at risk for HIV.

Our analyses also suggest that support for PrEP leads to knowledge of PrEP. We recognize that mediation analyses assume a directionality or causality between variables. ${ }^{18}$ However, it is also possible that knowledge of PrEP ultimately leads to increased support. The relationship between knowledge and support of PrEP is likely bidirectional, such that increased knowledge of PrEP is associated with increased support, and the converse is also true; these constructs are interrelated. We note, however, that it is possible for physicians to have knowledge of PrEP and yet not support PrEP prescription; our findings suggest that increased knowledge of PrEP does in fact lead to increased support. Future studies should use statistical modelling techniques that can account for such bidirectionality.

Lastly, the questions used for knowledge of PrEP, History of PrEP Prescription and barriers have not been previously validated by another study. Rather, they were developed specifically for this questionnaire. This calls into question the accuracy of these questions for measuring the outcomes they were intended to measure. Validating these items would be necessary to ensure the rigour of future findings.

\section{Conclusion}

This study elucidated the current state of Nova Scotian health care providers' support, knowledge, and attitudes towards PrEP. The sample of physicians in Nova Scotia in this study are largely aware of HIV PrEP and are supportive of its usage as a form of HIV prevention, but overall, do not feel knowledgeable enough to prescribe PrEP to their patients. Importantly, physicians recognized several barriers to prescribing PrEP, most notably the cost of the drug for patients. In turn, early introduction and the distribution of improved education materials as well as universal coverage for PrEP is expected to improve its integration and uptake in Nova Scotia.

\section{Data Sharing Statement}

Data available on request from the corresponding author.

\section{Ethics Approval}

The questionnaire and methodology for this study was approved by the Human Research Ethics board of Dalhousie University (REB Number: 2018-4481). Informed consent was obtained from all individual participants included in the study.

\section{Funding}

The study did not receive any funding.

\section{Disclosure}

Dr Darrell Tan reports grants from Canadian Institutes of Health Research/Ontario HIV Treatment Network, during the conduct of the study; grants from Gilead, investigatorinitiated research grant from Abbvie, and being a site principal investigator for industry-sponsored clinical trials for Glaxo Smith Kline, outside the submitted work. The authors declare that they have no other potential conflicts of interest for this work.

\section{References}

1. Fonner VA, Dalglish SL, Kennedy CE, et al. Effectiveness and safety of oral HIV preexposure prophylaxis for all populations. AIDS. 2016;30(12):1973-1983. doi:10.1097/QAD.0000000000001145

2. Khurana N, Yaylali E, Farnham PG, et al. Impact of improved HIV care and treatment on PrEP effectiveness in the United States, 2016-2020. JAIDS J Acquir Immune Defic Syndr. 2018;78 (4):399-405. doi:10.1097/QAI.0000000000001707 
3. Grant R, Lama J, Anderson P, et al. Preexposure chemoprophylaxis for HIV prevention in men who have sex with men NEJM. $N$ Engl $J$ Med. 2010;363(27):2587-2599. doi:10.1056/NEJMoa1011205

4. CADTH. HIV pre-exposure prophylaxis with emtricitabine/tenofovir disoproxil fumarate — regulatory and reimbursement policies; 2017. Available from: https://cadth.ca/dv/hiv-pre-exposure-prophylaxisemtricitabinetenofovir-disoproxil-fumarate-regulatory-and-

reimbursement. Accessed January 29, 2020.

5. Sharma M, Wilton J, Senn H, Fowler S, Tan DHS. Preparing for PrEP: perceptions and readiness of Canadian physicians for the implementation of HIV pre-exposure prophylaxis. PLoS One. 2014;9(8):e105283. doi:10.1371/journal.pone.0105283

6. Krakower DS, Mayer KH. The role of healthcare providers in the roll-out of PrEP. Curr Opin HIV AIDS. 2016;11(1):41-48. doi:10.1097/COH.0000000000000206

7. Adams LM, Balderson BH. HIV providers' likelihood to prescribe pre-exposure prophylaxis (PrEP) for HIV prevention differs by patient type: a short report. AIDS Care. 2016;28(9):1154-1158. doi:10.1080/09540121.2016.1153595

8. Karris MY, Beekmann SE, Mehta SR, Anderson CM, Polgreen PM. Are we prepped for preexposure prophylaxis (PrEP)? Provider opinions on the real-world use of PrEP in the United States and Canada. Clin Infect Dis. 2014;58(5):704-712. doi:10.1093/cid/cit796

9. Reyniers T, Vuylsteke B, Pirotte B, et al. Physicians' preparedness for pre-exposure prophylaxis: results of an online survey in Belgium. Sex Health. 2018;15(6):606-611. doi:10.1071/SH18072

10. Walsh JL, Petroll AE. Factors related to pre-exposure prophylaxis prescription by U.S. primary care physicians. Am J Prev Med. 2017;52(6):e165-e172. doi:10.1016/j.amepre.2017.01.025

11. Blumenthal J, Jain S, Krakower D, et al. Knowledge is power! Increased provider knowledge scores regarding pre-exposure prophylaxis (PrEP) are associated with higher rates of PrEP prescription and future intent to prescribe PrEP. AIDS Behav. 2015;19(5):802-810. doi:10.1007/s10461-015-0996-Z

12. Tripathi A, Ogbuanu C, Monger M, Gibson J, Duffus W. Preexposure prophylaxis for HIV infection: healthcare providers' knowledge, perception, and willingness to adopt future implementation in the southern US. South Med J. 2012;105(4):199-206. doi:10.1097/ SMJ.0b013e31824fla1b

13. Bernaix LW. Nurses" attitudes, subjective norms, and behavioral intentions toward support of breastfeeding mothers. J Hum Lact. 2000;16(3):201-209. doi:10.1177/089033440001600304

14. Godin G, Bélanger-Gravel A, Eccles M, Grimshaw J. Healthcare professionals“ intentions and behaviours: a systematic review of studies based on social cognitive theories. Implement Sci. 2008;3 (1):36. doi:10.1186/1748-5908-3-36

15. Herbert KE, Urmie JM, Newland BA, Farris KB. Prediction of pharmacist intention to provide medicare medication therapy management services using the theory of planned behavior. Res Soc Adm Pharm. 2006;2(3):299-314. doi:10.1016/j.sapharm.2006.02.008

16. Haddad N, Li J, Totten S, McGuire M. HIV in Canada-surveillance report, 2017. Can Commun Dis Rep. 2018;44(12):348-356. doi:10.14745/ccdr.v44i12a03

17. Government of Nova Scotia. Province marks HIV/AIDS awareness week and world AIDS day. News Releases; 2018. Available from: https://novascotia.ca/news/release/?id=20191128003. Accessed June $12,2020$.

18. Field A. Discovering Statistics Using SPSS. 2nd ed. London: Sage; 2005

19. Pett M, Lackey N, Sullivan J. Making Sense of Factor Analysis: The Use of Factor Analysis for Instrument Development in Health Care Research. Thousand Oaks, California: Sage; 2003.

20. O'Connor B. SPSS and SAS programs for determining the number of components using parallel analysis and Velicer's MAP test. Behav Res Methods Instrum Comput. 2000;32(3):396-402. doi:10.3758/ BF03200807
21. George D, Mallery P. SPSS for Windows Step by Step: A Simple Guide and Reference.11.0 Update. 4th ed. Boston: Allyn \& Bacon; 2003.

22. Hayes AF, Cai L. Using heteroskedasticity-consistent standard error estimators in OLS regression: an introduction and software implementation. Behav Res Methods. 2007;39(4):709-722. doi:10.3758/BF03192961

23. Myers R, Myers R. Classical and Modern Regression with Applications. Belmont, California: Duxbury press; 1990.

24. Tabachnick B, Fidell L. Using Multivariate Statistics. 6th ed. Pearson; 2013. Available from: https://www.pearson.com/us/highereducation/program/Tabachnick-Using-Multivariate-Statistics-6thEdition/PGM332849.html. Accessed February 4, 2020.

25. Iacobucci D. Mediation analysis and categorical variables: the final frontier. J Consum Psychol. 2012;22(4):582-594. doi:10.1016/j. jcps.2012.03.006

26. Tan DHS, Hull MW, Yoong D, et al. Canadian guideline on HIV pre-exposure prophylaxis and nonoccupational postexposure prophylaxis. CMAJ Can Med Assoc J. 2017;189(47):E1448-E1458. doi:10.1503/cmaj.170494

27. Petroll AE, Walsh JL, Owczarzak JL, et al. PrEP awareness, familiarity, comfort, and prescribing experience among us primary care providers and HIV specialists. AIDS Behav. 2017;21(5):1256-1267. doi:10.1007/s10461-016-1625-1

28. Weiser J, Garg S, Beer L, Skarbinski J. Prescribing of human immunodeficiency virus (HIV) pre-exposure prophylaxis by HIV medical providers in the United States, 2013-2014. Open Forum Infect Dis. 2017;4(1). doi:10.1093/ofid/ofx003

29. Hull M, Tan D. Setting the stage for expanding HIV pre-exposure prophylaxis use in Canada. Can Commun Dis Rep Releve Mal Transm Au Can. 2017;43(12):272-278. doi:10.14745/ccdr.v43i12a05

30. Kelly JA. Ten things we need to do to achieve the goals of the end the HIV epidemic plan for America. J Acquir Immune Defic Syndr. 2019;82(Suppl 2):S94-S98. doi:10.1097/QAI.0000000000002166

31. Nelson LE, McMahon JM, Leblanc NM, et al. Advancing the case for nurse practitioner-based models to accelerate scale-up of HIV pre-exposure prophylaxis. J Clin Nurs. 2019;28(1-2):351-361. doi:10.1111/jocn.14675

32. Sharma M, Chris A, Chan A, et al. Decentralizing the delivery of HIV pre-exposure prophylaxis (PrEP) through family physicians and sexual health clinic nurses: a dissemination and implementation study protocol. BMC Health Serv Res. 2018;18(1):513. doi:10.1186/s12913-018-3324-2

33. Patel RR, Chan PA, Harrison LC, et al. Missed opportunities to prescribe HIV pre-exposure prophylaxis by primary care providers in Saint Louis, Missouri. LGBT Health. 2018;5(4):250-256. doi:10.1089/lgbt.2017.0101

34. Lord R. Nova scotia doctor shortage forces ongoing emergency department closures. globalnews.ca; 2019. Available from: https:// globalnews.ca/news/5986086/nova-scotia-doctor-shortage-emergency -room-closures/. Accessed February 4, 2020.

35. Nova Scotia Health Authority. Need a family practice data; 2020. Available from: http://www.nshealth.ca/need-family-practice-data. Accessed February 4, 2020.

36. Schneidereit P. PAUL SCHNEIDEREIT: Nova Scotia's family doctor shortage deepens outside Halifax | local-perspectives | opinion. The Chronicle Herald; 2019. Available from: https:/www.thechronicleher ald.ca/opinion/local-perspectives/paul-schneidereit-doctor-shortagedeepens-outside-halifax-378622/. Accessed February 4, 2020.

37. Blackstock OJ, Patel VV, Felsen U, Park C, Jain S. Pre-exposure prophylaxis prescribing and retention in care among heterosexual women at a community-based comprehensive sexual health clinic. AIDS Care. 2017;29(7):866-869. doi:10.1080/09540121.2017.1286287

38. Krakower DS, Ware NC, Maloney KM, et al. Differing experiences with pre-exposure prophylaxis in boston among lesbian, gay, bisexual, and transgender specialists and generalists in primary care: implications for scale-up. AIDS Patient Care STDS. 2017;31 (7):297-304. doi:10.1089/apc.2017.0031 
39. Newman R, Katchi T, Karass M, et al. Enhancing HIV pre-exposure prophylaxis practices via an educational intervention. Am $J$ Ther. 2019;26(4):e462-e468. doi:10.1097/MJT.0000000000000773

40. Saberi P, Berrean B, Thomas S, Gandhi M, Scott H. A simple pre-exposure prophylaxis (PrEP) optimization intervention for health care providers prescribing PrEP: pilot study. JMIR Form Res. 2018;2 (1):e2. doi:10.2196/formative.8623

41. Calabrese SK, Magnus M, Mayer KH, et al. Putting PrEP into practice: lessons learned from early-adopting U.S. providers' firsthand experiences providing HIV pre-exposure prophylaxis and associated care. PLoS One. 2016;11(6):e0157324. doi:10.1371/journal. pone. 0157324

42. Krakower DS, Oldenburg CE, Mitty JA, et al. Knowledge, beliefs and practices regarding antiretroviral medications for HIV prevention: results from a survey of healthcare providers in New England. PLoS One. 2015;10(7):e0132398. doi:10.1371/journal.pone. 0132398

43. Mullins TLK, Zimet G, Lally M, Kahn JA. Adolescent human immunodeficiency virus care providers' attitudes toward the use of oral pre-exposure prophylaxis in youth. AIDS Patient Care STDS. 2016;30(7):339-348. doi:10.1089/apc.2016.0048

44. Mayer K, Chan P, Patel RR, Flash C, Krakower D. Evolving models and ongoing challenges for HIV preexposure prophylaxis implementation in the United States. J Acquir Immune Defic Syndr. 2018;77 (2):119-127. doi:10.1097/QAI.0000000000001579
45. Stinchcombe A, Wilson K. Lesian, gay, and bisexual (LGB) aging. In: Rania P, Wlfson C, Kirkland S, Griffith L, editors. The Canadian Longitudinal Study of Aging (CLSA) Report on Health and Aging in Canada, Findings from Baseline Data Collection 2010-2015. Hamilton, Ontario: Canadian Longitudinal Study of Aging; 2018:194-209.

46. Community Based Research Centre. PrEP costs and coverage. PrEP Nova Scotia; 2018. Available from: https://prepnovascotia.ca/prescrib ing-prep/prep-costs-and-coverage/. Accessed June 12, 2020.

47. Quon A. Advocates, NDP call for Nova Scotia to fund universal coverage of HIV prevention drug. Global News; 2019. Available from: https://globalnews.ca/news/6247947/universal-coverage-novascotia-hiv-prevention-drug/. Accessed June 12, 2020.

48. BC Centre for Excellence in HIV/AIDS. BC first province to declare the end of AIDS as an Epidemic. BC; 2019. Available from: http:// cfenet.ubc.ca/news/forecast/bc-first-province-declare-end-aidsepidemic. Accessed February 6, 2020.

49. Flanagan T. The Wealth of First Nations. Fraser Institute; 2019.

50. Silverman T, Schrimshaw E, Franks J, et al. Response rates of medical providers to internet surveys regarding their adoption of preexposure prophylaxis for HIV: methodological implications - Thomas B., Eric W. Schrimshaw, Julie Franks, Yael Hirsch-Moverman, Hugo Ortega, Wafaa M. El-Sadr, Paul W. Colson; 2018. Available from: https://jour nals.sagepub.com/doi/full/10.1177/2325958218798373?url_ver=Z39. 88-2003\&rfr_id=ori:rid:crossref.org\&rfr_dat $=$ cr_pub\%3dpubmed. Accessed February 6, 2020.

\section{Publish your work in this journal}

HIV/AIDS - Research and Palliative Care is an international, peerreviewed open-access journal focusing on advances in research in HIV, its clinical progression and management options including antiviral treatment, palliative care and public healthcare policies to control viral spread. The manuscript management system is completely online and includes a very quick and fair peer-review system, which is all easy to use. Visit http://www.dovepress.com/testimonials.php to read real quotes from published authors. 